Meta

Journal des traducteurs

Translators' Journal

\title{
La normalisation au service du traducteur
}

\section{Carmen Gómez et María Pinto}

Volume 46, numéro 3, septembre 2001

URI : https://id.erudit.org/iderudit/002940ar

DOI : https://doi.org/10.7202/002940ar

Aller au sommaire du numéro

Éditeur(s)

Les Presses de l'Université de Montréal

ISSN

0026-0452 (imprimé)

1492-1421 (numérique)

Découvrir la revue

Citer cet article

Gómez, C. \& Pinto, M. (2001). La normalisation au service du traducteur. Meta, 46(3), 564-579. https://doi.org/10.7202/002940ar

\section{Résumé de l'article}

Étant donné le rôle que la normalisation joue tout au long du processus de traduction, ce travail analyse les outils de normalisation utilisés par les traducteurs et les services de traduction. On insiste spécialement sur l'importance de maîtriser les techniques documentaires afin d'avoir accès facilement à l'information normative issue des organisations nationales et/ou internationales. Dans ce sens, on présente quelques sites Web fournissant de ressources normatives ainsi que quelques projets de recherche et de développement des normes en traduction.
Ce document est protégé par la loi sur le droit d'auteur. L'utilisation des services d’Érudit (y compris la reproduction) est assujettie à sa politique d'utilisation que vous pouvez consulter en ligne.

https://apropos.erudit.org/fr/usagers/politique-dutilisation/ 


\title{
La normalisation au service du traducteur
}

\author{
CARMEN GÓMEZ \\ Université de Málaga, Málaga, Espagne \\ MARÍA PINTO \\ Université de Granade, Grenade, Espagne
}

\begin{abstract}
RÉSUMÉ
Étant donné le rôle que la normalisation joue tout au long du processus de traduction, ce travail analyse les outils de normalisation utilisés par les traducteurs et les services de traduction. On insiste spécialement sur l'importance de maîtriser les techniques documentaires afin d'avoir accès facilement à l'information normative issue des organisations nationales et/ou internationales. Dans ce sens, on présente quelques sites Web fournissant de ressources normatives ainsi que quelques projets de recherche et de développement des normes en traduction.
\end{abstract}

\begin{abstract}
Due to the role that normalization plays during translation processes, this work analyzes the standardization tools used by translators and translating services. Emphasis is placed on the importance of having a good command of documentary techniques in order to access easily the standardization information devised by national and international organizations. With this aim, web site URLs which include standardization resources are supplied, as well as some research and development of translation standards projects.
\end{abstract}

\section{MOTS-CLÉS/KEYWORDS}

documentation normative, normalisation, processus de traduction, sites Web, techniques documentaires

\section{Introduction}

La normalisation s'impose à tous les niveaux des domaines scientifiques et techniques, et ainsi la traduction ne peut pas rester à l'écart. Les avantages de la normalisation sont évidents et connus des traducteurs professionnels qui offrent un niveau élevé de qualité, d'efficacité et de fiabilité dans un marché de plus en plus global et hautement concurrentiel.

L'information normative devient donc un outil indispensable par:

a) la fiabilité du résultat du produit ou de l'activité normalisée;

b) la simplicité: les normes réduisent le nombre des variétés et unifient la terminologie et les élements des produits, leurs matériaux et leurs modes opératoires;

c) l'économie: les normes réduisent les coûts de production, de planification, des procédés, des techniques, etc.;

d) la coopération: les normes stimulent la coopération aux niveaux national et international et assurent l'interchangeabilité;

e) la pluridisciplinarité: les normes traitent de tous les domaines techniques, économiques et sociaux de l'activité humaine;

f) l'actualité: les normes suivent l'évolution du progrès technique et social et sont vérifiées périodiquement quant à leur validité ou selon les nécessités des utilisateurs; 
g) la qualité: les normes déterminent les critères, les méthodes et les procédures ainsi que le contrôle et l'évaluation des aspects normalisés.

La normalisation est une activité collective visant à trouver des solutions pour des situations répétitives par le biais des normes. Toute norme est conçue comme un ensemble de spécifications techniques qui doivent être respectées afin de garantir le plus haut niveau de qualité des activités et des produits. Elle est élaborée en collaboration et avec le consensus de tous les intéressés et elle est approuvée par un organisme qualifié sur le plan national, régional ou international.

La classification des normes respecte les critères suivants:

a) Le type de sujet normalisé: si la norme établit les concepts, les valeurs, les catégories, etc., on parle de normes générales. Si, par contre, la norme précise les conditions requises par les produits, ce sont les normes des produits; et finalement, il y a les normes concernant les activités qui définissent les méthodes et les procédures.

b) Le domaine d'application des normes: il y a les normes internationales produites par des organismes internationaux de normalisation; les normes régionales, élaborées par des intérêts régionaux; les normes nationales, s'il s'agit d'une organisation nationale, et finalement, les normes d'entreprises qui concernent exclusivement une entreprise.

c) Selon le ressort de l'organisme de normalisation: on distingue les normes de jure des normes de facto. Ainsi, une norme de jure (ou formelle) désigne une norme qui a été établie par un organisme légalement (ou formellement) constitué et mandaté pour élaborer et développer des normes. Au niveau international, il y a l'Organisation internationale de normalisation (ISO), et au niveau national, chaque pays a son organisme de normalisation. Citons, par exemple, l'Association française de normalisation (AFNOR) en France, ou le Conseil canadien de normalisation (CCN) au Canada. Par contre, une norme de facto désigne une norme qui a été élaborée par une organisation autre qu'un organisme formel de normalisation. Ainsi, par exemple, un consortium des fabricants d'ordinateurs, une association profesionnelle, une grande entreprise informatique, etc.

d) L'aspect de la normalisation: on distingue alors les normes de terminologie et de définitions des concepts, les normes contenant des spécifications techniques sur les produits et les normes sur les procédures.

e) On connaît également les normes obligatoires, qu'il faut absolument observer, et les normes facultatives ou recommandées.

\section{Le rôle des organisations de normalisation dans le domaine scientifique et technique}

L'importance des normes élaborées par les organisations de normalisation constituées formellement obligent les traducteurs à connaître la voie d'accès à cette information. Afin d'esquisser un panorama de ces organisations, nous allons les présenter et les classer selon leur domaine d'application.

\subsection{Organisations Internationales de Normalisation}

L'organisation de normalisation la plus importante est l'ISO, créée en 1947 pour développer des normes universelles dans le but d'améliorer la communication et la coopération internationales dans les domaines scientifique, technique et économique. L'ISO regroupe environ une centaine d'organismes nationaux de normalisation et elle est constituée de plus de 800 comités techniques ou sous-comités, chargés de l'élaboration des normes. Cependant, les normes qui concernent les secteurs de 
l'électrotechnique et des télecommunications ressortissent à la Commission électrotechnique internationale $(\mathrm{CEI})$ et à l'Union internationale des télécommunications (UIT).

Les normes ISO représentent le meilleur consensus possible de toutes les parties concernées, et de ce fait, elles sont les plus réputées et appréciées, ainsi que largement applicables au niveau mondial. Elles sont généralement publiées en deux versions séparées: l'anglaise et la française. Certaines normes, notamment dans le domaine de la terminologie, sont publiées en édition bilingue (anglais/français) ou trilingue (anglais/français/russe).

Les normes internationales ISO contiennent les éléments suivants :

- $\quad$ un numéro de référence constitué d'un préfixe qui identifie l'organisation;

- un numéro de série et l'année de publication;

- le titre de la norme suivi de l'identifiant ICS (Classification internationale pour les normes, classification hiérarchique à trois niveaux);

- un ensemble de descripteurs attribués à chaque document afin de faciliter la recherche des normes par sujet. Les descripteurs font partie du Thésaurus international technique (TIT), utilisé par l'ISO afin d'indexer les documents et dont l'auteur est l'AFNOR.

Pour faciliter l'accès à l'information des normes, l'ISO a créé ISONET, un réseau mondial d'information formé de nombreux centres nationaux d'information qui fournissent des renseignements de caractère normatif tels que les normes, les recommandations, les rapports techniques, les systèmes de certifications, etc. Ainsi, ces points d'information spécialisés diffusent l'information sous la forme de différentes publications: catalogues, bulletins, brochures d'information, accès aux bases des données, etc. Le réseau Internet est une autre possibilité pour le repérage d'information fournie par l'ISO.

Le catalogue ISO, par exemple, permet la recherche des normes par sujets, par les mots du titre, par descripteurs et par le numéro de réference ISO. L'information fournie présente les élements suivants:

- la notation ICS du groupe ou sous-groupe dans lequel la norme donnée est classée;

- des abréviations indiquant le comité technique responsable de son élaboration;

- des informations concernant le code du prix et des descripteurs du contenu de la norme donnée.

\subsection{Les organisations régionales de normalisation}

Ce sont des organismes qui ont des activités reconnues dans le domaine de la normalisation au niveau régional. Ils sont ouverts à tout organisme national représentant un pays à l'intérieur d'une seule et même zone géographique, politique ou économique. Ils sont généralement intégrés à l'ISO. Nous pouvons citer le Comité européen de normalisation (CEN), fondé en 1961, dans le but de développer des activités de normalisation au niveau européen pour favoriser les échanges des produits et des services. Les États membres de l'Union européenne en font partie. Le champ d'action du CEN couvre tous les domaines de la normalisation, à l'exception de la technologie de l'électronique et de la communication, qui sont du ressort du Comité européen de la normalisation électrotechnique (CENELEC) depuis 1959. De son côté, l'Institut européen des normes de télécommunication (ETSI), fondé en 1988, est constitué par des associations et des entreprises européennes pour ce même secteur. 
Les résultats des activités des organismes européens de normalisation sont les normes européennes (EN), des documents harmonisés et des normes expérimentales. Les normes européennes sont systématiquement reprises, sans aucune modification, comme normes nationales dans tous les pays de l'Espace économique européen, avec suppression obligatoire des normes nationales divergentes. Par contre, les normes internationales sont reprises comme normes nationales de manière facultative, avec ou sans modification.

\subsection{Les organismes de normalisation nationaux}

Ce sont des organismes qui ont des activités reconnues dans le domaine de la normalisation au niveau national et dont l'une des principales fonctions est la préparation, l'approbation ou l'adoption de normes mises à la disposition du public de plusieurs façons.

En France, l'organisme de normalisation est AFNOR ([http://www.afnor.fr]), placé sous la tutelle du ministère chargé de l'Industrie, dont l'une des missions essentielles est de promouvoir et de faciliter l'utilisation des normes. Dans ce sens, l'AFNOR met au point des produits et des services adaptés aux besoins d'entreprises et de collectivités tels que des stages de formation, des prestations de conseil, l'édition de différentes publications qui garantissent ainsi l'accès à l'information :

- le Catalogue AFNOR répertorie les 20000 normes françaises, européennes et internationales en vigueur;

- $\quad$ une base des données bibliographiques sur cédérom (PERINORM) répertorie les normes et les projets de normes de nombreux pays;

- une base des données bibliographiques sur Minitel;

De son côté, le Conseil canadien des normes (CCN) est chargé de représenter le Canada au sein de deux grands organismes internationaux de normalisation: l'ISO et la CEI, mais il veille aussi aux travaux de normalisation au Canada, entérinant les normes nationales. Le CCN est responsable de la coordination des activités de normalisation entreprises au Canada par cinq organisations accréditées. Parmi elles, l'Association canadienne de normalisation (CSA), qui se consacre particulièrement à l'élaboration de normes canadiennes dans le domaine des technologies de l'information. Le CCN est la source principale de renseignements au Canada en matière de normalisation. Son site Web (<http://www.ccn.ca $>$ ) offre aux visiteurs l'information la plus récente sur les normes, les règlements techniques et les activités d'évaluation de la conformité. De plus, le CCN gère un service d'information et de recherche qui contient différentes bases de données avec un accès détaillé et complet à l'information sur les normes. Il publie également CONSENSUS, une revue d'actualité consacrée à la normalisation, et sert de centre de distribution des normes étrangères et internationales.

\section{Les procès de la traduction et la normalisation}

Dans cette section, nous analysons les outils de normalisation utilisés par les traducteurs et les services de traduction tout au long de leur travail. On peut ainsi distinguer les normes, mais aussi les conseils, les recommandations et les instructions utilisés par les traducteurs. La différence se situe dans le mode de conditionnement 
de la conduite du traducteur. Ainsi, si les conseils se bornent simplement à faire des recommandations, les instructions fixent aux traducteurs d'un service déterminé des conditions précises. Et enfin, les normes doivent être absolument suivies par tous les traducteurs (Pinto 1993: 112).

Parmi les instructions, citons celles utilisées par le Service de traduction de la Commission européenne dans le cadre d'EURODICAUTON, base de données terminologique multilingue (<http://www2.echo.lu/eurod $>$ ). Quant aux normes, les entreprises et les services de traduction adoptent de plus en plus les normes de qualité ISO 9000 et DIN 2345 (voir paragrahe 2.1), cherchant à assurer ainsi la qualité de leurs produits et de leurs services, tout en bénéficiant du logo publicitaire. C'est le cas de l'agence de traduction nord-américaine ASET (<http://www. asetquality.co $>$ ) qui compte parmi ses clients le ministère de la Défense des États-Unis et la NASA.

D'un autre côté, il faut insister sur le fait que le traducteur est la clé du processus de traduction par sa qualité d'auteur d'un document nouveau conforme au texte source. Même si son niveau de connaissances est très élevé, il reste cependant insuffisant pour achever son travail. Il doit donc utiliser les techniques de la documentation, qui sont des outils indispensables pour sélectionner et analyser l'information repérée sur le sujet à traiter. Ces techniques documentaires vont aussi être utiles ensuite dans le procès technique de la traduction ainsi que dans l'élaboration du texte meta. La compétence linguistique, cognitive, scientifique et fonctionnelle du traducteur devra être complétée par son habileté dans les techniques de la documentation, ce qui lui permettra non seulement une plus grande organisation de son travail, mais aussi un accès plus rapide aux documents et au repérage de l'information voulue.

Les théoriciens de la traduction distinguent généralement deux concepts:

- d'un côté, l'activité traductionnelle ou le processus de traduction;

- de l'autre côté, le résultat ou le produit de cette activité.

Étant donné cette distinction, la normalisation semble concerner plus directement le produit de la traduction, surtout si l'on pense aux aspects de la présentation des résultats. Par contre, les normes sont en rapport avec les processus de traduction en général, depuis le début. Par exemple, c'est le cas des contrats de traduction inclus par Peña et Hernandez Guerrero (1994: 72) dans une première phase de prétraduction, selon le schème des processus de traduction qu'ils proposent. Et de même, d'autres aspects tels que ceux qui concernent le vocabulaire ou les méthodes de traduction, que l'on traitera plus loin.

\subsection{Les contrats de traduction}

Le Deutsches Institut für Normung a publié la norme DIN 2345 qui est sur le point d'être acceptée comme norme européene. Elle couvre tous les aspects traitant de la régularisation des contrats entre client et traducteur: temps de remise, délais, droits d'auteur, prix, etc. Une telle procédure contribuera à accroître certainement la fiabilité des deux parties.

En fait, cette norme DIN 2345 assure la qualité des produits de la traduction, s'ajoutant ainsi aux normes de qualité ISO 9000 (Sturz 1998).

Pour sa part, l'Organisation italienne pour l'assurance de la qualité (UNITER) a aussi publié la norme UNI 10574 (Definizzione dei serzi e della attivita delle imprese 
di trauzione ed interpretariato), approuvée par l'Institut italien de normalisation, qui définit les services offerts par les agences de traduction et d'interprétariat.

En plus de ces normes nationales, d'autres organisations, telles que l'Association des traducteurs et interprètes de l'Ontario $\left(\mathrm{ATIO}^{1}\right)$, ont publié des recommandations pour régulariser l'activité des traducteurs professionnels. La page Web d'ATIO (<http://www.atio.on.ca $>$ ) offre un modèle de contrat pour ses membres et, en général, pour tous les traducteurs.

\subsection{Le vocabulaire et la terminologie}

C'est dans ce domaine du vocabulaire et de la terminologie que la normalisation atteint toute son importance. Les normes sont en effet de précieux outils dans les échanges nationaux et internationaux, tout comme pour l'industrie de la gestion de l'information et l'interopérabilité technologique.

Ainsi la terminologie est-elle devenue une priorité de prime importance dans les efforts de normalisation des organisations nationales et internationales. L'ISO élabore des normes dans le domaine de la terminologie depuis plus de 40 ans avec l'intention de relever le défi que lui pose l'adaptation des produits aux besoins actuels, ainsi qu'aux nouveaux besoins qui voient le jour. Ces normes ISO concernent l'établissement de vocabulaires techniques dans tous les domaines de spécialisation: scientifique, intellectuel, technologique et économique. Dans la section 01.040 Vocabulaires du catalogue ISO, ces normes se répartissent en 30 grands domaines de spécialité. L'ISO s'occupe de même de la normalisation des principes et des méthodes en terminologie et terminographie pour assurer la coopération et la coordination avec d'autres organisations concernées par l'activité terminologique. Le comité technique ISO TC37 (<http://www.ssc.ca/surreys.isofr.htm>) mène actuellement une analyse de besoins et de clientèle dont l'objectif est de fournir aux normalisateurs techniques et terminologiques - et, dans une plus large mesure, à tous les établissements et à toutes les organisations engagées dans la création et le traitement des ressources linguistiques et de l'information spécialisée - des normes, des directives et des outils informatisés qui leur permettent d'utiliser les méthodologies pertinentes à des fins d'élaboration de normes de qualité pour une mélioration de l'efficacité du travail terminologique et de la qualité des terminologies produites.

L'ISO a également développé des formats d'échange de données terminologiques (ISO 6156 et ISO 2709) afin de rendre possible la compatibilité entre les banques de données déjà existantes, et ensuite entamer la normalisation technique des produits.

La normalisation du vocabulaire spécialisé est indispensable et pour la représentation des connaissances et leur transmission. Au fur et à mesure qu'une langue se spécialise, son lexique devient plus concret. Elle a donc besoin de préciser les dénominations afin de conceptualiser adéquatement la réalité. On sait que les processus de la normalisation terminologique sont complexes: ils comprennent plusieurs étapes:

- l'unification des concepts et des systèmes des concepts;

- la définition des termes;

- l'élimination de la synonymie;

- la réduction de l'homonymie;

- l'établissement des abréviations et des symboles, etc. 
Il ne faut pas non plus oublier l'automatisation du traitement des données terminologiques. Le stockage des textes complets sous format optique rend possible leur transfert dans des formats appropiés à l'analyse terminologique. En utilisant les techniques de la linguistique computationnelle, on réussit à obtenir des listes des termes nouveaux ou des termes équivalents dans d'autres langues.

La plupart des banques terminologiques visent les unités lexicalisées, c'est-àdire, des termes simples ou composés (NORMATERM). D'autres banques de données, telles que EURODICAUTOM, acceptent aussi les phrases et les propositions.

Finalement, la technologie de l'hypertexte dans le domaine des banques des données terminologiques aide l'établissement de nouvelles relations conceptuelles non identifiées au moment de la compilation. Étant donné les caractéristiques particulières des structures hypertextuelles, celles-ci permettent de créer de nombreuses relations entre les nœuds d'informations, qui les relient de manières différentes. Les définitions des termes vont s'enrichir ainsi, et l'utilisateur sera orienté vers l'information la plus complète: l'information personnalisée. Les nouvelles structures terminologiques présentent de grands avantages du fait qu'elles permettent la représentation de structures conceptuelles de n'importe quel sujet à l'intérieur d'une banque de données.

Outre les organismes de normalisation et de standardisation, d'autres organisations contribuent à l'harmonisation de nouvelles terminologies au sein de la francophonie, en favorisant la coopération internationale en matière d'aménagement linguistique. Il s'agit du RINT, le Réseau international de néologie et de terminologie, une organisation intergouvernementale francophone créée en $1986(<\mathrm{http}: / /$ www. rint.org $>$ ). Les réalisations du RINT sont nombreuses et variées: publication de travaux terminologiques, élaboration d'outils informatisés pour le travail terminologique, inventaire des ressources terminologiques et terminotiques, mise à jour des banques de terminologie et des dictionnaires spécialisés, etc.

Une autre instance qui s'intéresse à la normalisation de la terminologie est l'Office de la langue française (OLF) (<http://www.olf.qc.ca $>$ ), organisation gouvernementale chargée de promouvoir la langue française et de définir et de conduire la politique québécoise en matière de linguistique et de terminologie. L'OLF officialise ses choix linguistiques au moyen d'avis de normalisation ou de recommandations.

Étant donné le grand nombre d'institutions et de personnes qui travaillent en normalisation, le Centre francophone d'expertise de veille Inforoutes et Langues (CEVEIL) (<http://www.ceveil.qc.ca $>$ ), a produit une liste qui peut être complétée avec un inventaire à jour des membres de tous les comités de terminologie internationaux et nationaux ainsi que de ceux des comités de terminologie dans les services linguistiques des diverses entreprises de la francophonie.

D'autre part, la plupart des organisations publiques et privées publient les résultats de leurs activités sous la forme de dictionnaires, de vocabulaires, de lexiques, de thésaurus et, de plus en plus, de banques de données terminologiques informatisées. C'est le cas du Bureau de la traduction de la direction de la terminologie et de la normalisation (DTN) du gouvernement fédéral du Canada, qui contribue activement aux travaux de normalisation en publiant des vocabulaires normalisés dans le cadre de l'administration fédérale canadienne (élaboration du Lexique de la norme générale de classification ${ }^{2}$ ) et en offrant des produits tels que TERMIUM. Il s'agit d'un dictionnaire électronique anglais-français réunissant plus de trois millions de 
termes et d'appellations, ainsi que des définitions, des informations grammaticales et stylistiques pour la rédaction, équivalentes dans les deux langues, des abréviations, des exemples d'utilisations et des observations dans presque tous les domaines du savoir.

Dans cette perspective, il faut mentionner aussi TERMISTI, une institution universitaire francophone, rattachée à l'Institut supérieur de traducteurs et interprètes (ISTI, Bruxelles). Ses activités portent essentiellement sur la description terminologique et la terminotique, et parmi les plus intéressantes, nous pouvons citer la production de glossaires terminologiques concernant les domaines de haute spécialité.

\subsection{La normalisation et les technologies de l'information}

Les nombreuses possibilités fournies par les nouvelles technologies telles que la capacité de stockage des disques cédérom et la présence de divers protocoles de communication et d'interconnexion fournis par Internet ont fait accroître les efforts d'élaboration de nouvelles normes et recommandations en technologie de l'information (normes TI).

Ce sont l'ISO, le réseau international Internet, le groupe international TEI (Initiative pour la codification des textes), ainsi que le groupe EAGLES (Expert Advisory Group on Language Engineering Standards) de la Commisssion européenne, organisations chargées de produire les normes TI. À l'exception de l'ISO, toutes les autres organisations élaborent des normes de facto qui généralement vont devenir des normes internationales de jure.

L'organisation internationale Internet est responsable de la coopération et de la coordination des technologies et des applications Internet à travers le monde. Les normes de facto/recommandations Internet sont accessibles gratuitement sur le Web et identifiables par l'abréviation RFC (Request for Comment). Il s'agit de normes techniques relatives aux divers protocoles de communication, de transmission, de transfert et d'interconnexion du réseau Internet.

Le groupe TEI (Text Encoding Initiative) a été constitué en 1987 lors de l'union de l'association américaine ACH (Association for Computer and the Humanities) et des associations internationales ACL (Association for Computational Linguistics) et ALLC (Association for Literary and Linguistic Computing). D'autres organismes publics, tels que la Direction générale DGXIII de la Commission des communautés européennes, ont, par la suite, participé aux travaux du groupe TEI. Le résultat de cette collaboration a été la normalisation des points suivants qui concernent directement les aspects technologiques de la traduction:

a) l'échange de données terminologiques entre diverses organisations, divers systèmes et divers outils logiciels de façon à rendre possible une véritable transaction d'information, même s'il existe des différences techniques. Les normes les plus importantes dans ce domaine sont:

- la norme ISO 8879:1986 ou SGML (Langage normalisé de balisage généralisé), un modèle ouvert permettant la production de documents électroniques;

- la norme de facto TEI P3, qui définit un format standard d'encodage de textes électroniques; et finalement,

- la norme MARTIF (ISO 12200) sur l'échange de terminologie, qui a été créée en collaboration avec TEI et LISA (Localisation Standards Association).

b) l'encodage des caractères pour le traitement informatisé des données multilingues. On y trouve de nombreuses normes, telles que la norme ISO 646 ou la norme UNICODE 
sur les jeux de caractères codés. De plus, il convient de citer les travaux du comité européen CEN/TC304, dont les résultats seront concrétisés en de nouvelles normes pour le traitement informatique des langues européennes.

Dans le contexte francophone, l'intérêt pour la normalisation des technologies de l'information est devenu une priorité pour de nombreuses organisations:

- RIOFIL (Réseau international des observatoires francophones de l'inforoute et du traitement informatique des langues) : réseau composé par la France, le Canada, le Québec, la Suisse, la Wallonie et l'Afrique. Parmi ses activités, le RIOFIL envisage de favoriser la diffusion et l'utilisation des normes et des standards au profit de la diversité linguistique et culturelle dans les technologies de l'information.

- CEVEIL (Centre d'expertise de veille Inforoutes et Langues) (<http://www.ceveil.qc.ca $>$ ): organisme à but non lucratif qui existe depuis 1995. Créé par le gouvernement du Québec afin de mieux cerner la problématique des langues sur les inforoutes dans le cadre francophone. Parmi ses objectifs, CEVEIL vise à mettre en évidence le plurilinguisme et l'importance des normes et des standards dans le contexte des technologies de l' information.

- NoTIAL: groupe de travail franco-québécois sur la normalisation des technologies de l'information dans leurs aspects linguistiques (<http://www.ceveil.qc.ca/notial/mandat. html $>$ ). Son objectif est d'identifier les normes et les standards permettant d'accéder, en français, aux autoroutes de l'information afin d'arriver à une réelle concertation franco-québécoise relative à la mise en vigueur des normes et des standards utiles au traitement de l'ensemble des langues de l'humanité par la voie informatique.

\subsection{L'analyse de contenu. Outils documentaires du traducteur}

Dans le domaine des activités documentaires, il convient de signaler que le traducteur est celui qui traite l'information repérée et celui qui produit un nouveau document. C'est lui qui convertit l'analyse du contenu, plus précisément l'analyse textuelle et pragmatique, en l'une des opérations principales pour arriver finalement à un texte cohérent et riche de signification. La difficulté de cette opération se trouve dans:

- le caractère intellectuel et créatif de la sélection et de la représentation du contenu des documents, qui se situe à la limite du langage et du cognitif;

- les difficultés posées par le langage des documents et leur signifié;

- des questions strictement documentaires relatives à l'analyse et à sa profondeur, au caractère hétérogène et subjectif des analystes, à la diversité des structures et des contenus des documents, aux contextes multiples et aux différents besoins des usagers.

Dans le paragraphe suivant, nous insisterons sur les normes internationales, qui peuvent aider le traducteur à comprendre et à améliorer les procédures documentaires.

La norme ISO 5963-85 incite les services d'indexation et d'autres centres de documentation à unifier leurs pratiques en ce qui concerne l'indexation humaine. La norme définit les concepts et insiste sur la méthodologie de l'indexation, tout en présentant des recommandations pour les étapes suivantes:

- l'analyse du document pour repérer les identifiants de l'information significative;

- la sélection des concepts fondamentaux;

- la sélection des termes d'indexation dont la présentation et la structure vont dépendre du système choisi. 
D'un côté, la normalisation des langages d'indexation vise l'amélioration de la terminologie scientifique et technique dans tous les domaines, en publiant des dictionnaires, des lexiques et des glossaires, outils de plus en plus nécessaires pour l'établissement et la validation des langues spécialisées. Dans ce sens, les efforts de l'UNESCO sont remarquables, et spécialement ceux de l'ISO, qui déploie une grande partie de son activité dans le cadre du Comité technique 37 ou dans celui des normes nationales reliées au vocabulaire de documentation et information.

D'un autre côté, on ne peut oublier la normalisation du langage documentaire ou langage contrôlé, de plus en plus utilisé pour la représentation formelle des concepts des documents analysés. Il y a deux normes importantes relatives à l'élaboration de thésaurus: ISO 2788:1986 et ISO 5964:1985. Il faut citer aussi la norme française NF Z47-100: 1981 pour les thésaurus monolingues et la norme NF Z47-101: 1990 pour les thésaurus multilingues, équivalentes aux normes ISO. Elles traitent des questions telles que la définition des concepts et des méthodes pour contrôler le vocabulaire, en signalant la forme et la structure des termes d'indexation ainsi que les relations qu'ils entretiennent dans le thésaurus et les différentes présentations que ces derniers peuvent adopter.

Par ailleurs, la norme ISO 5964:1985 présente les difficultés dans la construction d'un thésaurus multilingue relevant aussi bien de la stratégie (configuration, prise des décisions sur le niveau accordé à une des langues du thésaurus...) que de la technique. La norme insiste sur les aspects de l'élaboration du thésaurus, qui peut être réalisé sans consulter d'autres thésaurus spécialisés dans la même matière, ou bien, qui peut être une traduction en diverses langues à partir d'une première langue, ou encore une adaptation d'autres thésaurus en langues différentes ce qui devient une tâche difficile étant donné l'absence de correspondance entre les structures logiques et les matières couvertes.

Cette norme insiste spécialement sur les équivalences des termes d'indexation en langues différentes, et analyse les difficultés posées par la traduction. La forme, le contenu et la présentation de ce thésaurus sont également mis en évidence. La présentation renferme une grande richesse: des thésaurus dont les termes sont classés thématiquement selon l'ordre alphabétique d'une des langues, des thésaurus bilingues et trilingues. Parmi les thésaurus multilingues élaborés selon la norme internationale, il faut mentionner l'AGROVOC, le Thésaurus agricole multilingue. Cette norme est en général un important outil pour le traducteur qui veut connaître le vocabulaire contrôlé (descripteurs) d'une matière déterminée, ainsi que les équivalents dans d'autres langues, dans le but de permettre la compatibilité entre les langues et les langages (langage naturel du traducteur et de l'utilisateur / documentaire du système d'information). La norme ISO 5964:1985 joue un rôle important dans l'amélioration du contrôle bibliographique et dans les systèmes de récupération multilingues.

Finalement, citons la norme française NF Z44-004-1984, équivalente à la norme ISO 214:1976, qui assure un cadre suffisamment large pour réunir les différentes méthodes d'élaboration de résumés, se basant sur un modèle multiparadigmatique, consolidé par la linguistique textuelle, le traitement de l'information, les modèles logiques et communicatifs. À la suite d'une brève définition, cette norme présente les fonctions du résumé et leur typologie en insistant sur des aspects stylistiques et sémantiques généraux qui touchent plus la forme et la présentation du résumé que son contenu. 


\subsection{Quelques méthodes de traduction}

Dans ce paragraphe, nous signalerons les normes existantes relatives à quelques outils du traducteur. Nous faisons référence surtout aux mémoires de traduction et aux logiciels d'aide à la traduction et à l'évaluation des systèmes de TAO (Traduction assistée par ordinateur). Pour une information plus détaillée, on peut consulter le document de travail de CEVEIL (Bourbeau 1995). Les mémoires de traduction sont construits à partir de textes déjà traduits et constituent une aide aussi bien pour traduire des textes repétitifs que pour faire des recherches terminologiques. Il n'existe pas encore de normes de jure pour l'élaboration et la gestion de mémoires de traduction, mais plutôt des méthodes et des techniques faisant partie du domaine de la gestion documentaire et des techniques de l'indexation. C'est pour cette raison qu'il faut tenir compte des normes concernant ce sujet précis. On peut diviser les mémoires de traduction en deux grandes classes: les mémoires construits à partir d'une indexation de phrases complètes et les mémoires construits à partir d'une indexation de tous les mots. La méthode de recherche textuelle sera donc déterminée par la technique d'indexation utilisée.

De son côté, l'organisation LISA étudie une recommandation TXM (Translation Memory Exchange) qui vise à faciliter l'échange des données des mémoires de traduction entre outils et logiciels d'aide à la traduction.

Pour l'évaluation de la qualité des logiciels d'aide à la traduction, nous avons la norme ISO/IEC 9126:1991. La norme ISO/IEC définit les exigences de qualité, les méthodes d'évaluation et l'application des procédures d'évaluation. Dans ce sens, il convient de citer les travaux du groupe EAGLES, relatifs aux dictionnaires électroniques multilingues, les thésaurus électroniques multilingues, les systèmes de gestion de données terminologiques et les mémoires de traduction.

Mais le groupe EAGLES ne vise pas à produire des normes internationales mais de présenter les besoins et les exigences d'applications opérationnelles et d'accélérer le processus de normalisation dans cette matière.

Il n'y a pas non plus de normes spécifiques pour la traduction automatique. Toutefois, les systèmes de TAO ont été soumis à de nombreuses évaluations permettant ainsi d'améliorer progressivement les méthodologies d'évaluation. C'est la raison pour laquelle certaines de ces évaluations peuvent être considérées comme étant des normes de facto pour l'évaluation des technologies TAO.

\subsection{La présentation des traductions}

C’est à partir du résultat ou le produit de la traduction que nous analyserons quelques aspects de la normalisation concernant la caractérisation de tous les documents issus de la traduction d'œuvres originales. Il s'agit de la norme NF Z 41-004: 1979, équivalente à la norme ISO 2384 : 1977 . Elle n'aborde pas les détails matériels pour la présentation du texte, tels que la composition, le format, les marges, etc., mais les caractéristiques qui doivent faciliter l'identification des traductions et celle des œuvres dont elles procèdent. Les questions envisagées sont les suivantes:

a) Éléments d'identification à inclure dans la présentation d'une traduction, aussi bien ceux qui sont relatifs à la traduction que ceux qui concernent le document original. Évidemment, ces données vont être différentes selon la typologie documentaire, ce qui 
renvoie aux normes de description et à d'autres normes pour la présentation de symboles, d'unités, de noms de pays et de langues.

b) La structure de la traduction.

c) Les notes, les notes en bas de page et les références bibliographiques; les formules et les équations, ainsi que les figures, les légendes et les titres de figures et de tableaux.

d) La translittération; les noms et les sigles d'organismes, les abréviations, la terminologie, les noms géographiques et les dates.

\section{Sources d'information sur les normes pour les traducteurs}

Pour un traducteur, le fait de se documenter est une phase importante pour réaliser son travail, dans la mesure où il va obtenir des connaissances linguistiques et extralinguistiques fondamentales pour comprendre un texte et pour présenter ensuite la langue meta (Hurtado Albir 1996). Dans ce sens, les normes constituent une source de premier ordre pour les traducteurs, car elles vont fournir, en tant que documents contenant des spécifications techniques (symboles, définitions, codes, figures, méthodologie, etc.), une information abondante, exacte et fiable.

Le fait que les normes soient de plus en plus nombreuses dans tous les domaines, et surtout en traduction, exige une activité de normalisation parallèle des normes afin de rendre plus faciles leur repérage et leur identification. Cette activité constitue l'objet de la Documentation sur les normes ou sur l'ensemble organisé des normes (Pinto 1993: 113). Dans la mesure où les textes, objets de traduction, présentent une grande diversité thématique, l'activité documentaire appliquée aux normes peut guider et orienter le traducteur dans la recherche de normes relatives à un domaine déterminé. La production de standards est devenue parfois si prolifique qu'il est bien difficile de s'y retrouver. Les techniques documentaires apportent une aide indispensable. Les services d'information et de diffusion des organismes de normalisation nationaux et internationaux permettent l'accès à toute cette information. Cependant, il ne faut pas oublier les services de recherche documentaire fournis par des organismes privés, tels PRODOC, une équipe internationale d'ingénieurs spécialistes dans l'élaboration de la documentation technique dans toutes les langues européennes et dans d'autres langues. PRODOC assure une bonne traduction en remplissant toutes les formalités, y compris la recherche dans des banques de données des normes et des directives européennes applicables aux produits (<http://www.prodoc.de $>$ ).

\subsection{Projets de recherche et de développement des normes en traduction}

L'effort mené pour la normalisation est global et mondial. On le ressent dans tous les pays et dans tous les domaines, d'autant plus que l'on vit une époque de mondialisation de la communication et des technologies nouvelles (marché informatique, l'autoroute électronique, etc.). Le besoin de normaliser les systèmes, les méthodes, les outils, les concepts, etc., devient une exigence en vue de la communication mondiale et des échanges internationaux. Citons quelques exemples qui peuvent intéresser les traducteurs:

a) Projets de la Direction générale XIII de la Commission européenne relatifs à la normalisation et à l'évaluation de ressources, d'applications et de services dans le cadre de l'ingénierie linguistique: <http://www.tagish.co.uk/thos/tap/tappoj.htm> 


\section{a.1. Programme MLIS}

(<http://www.info2000.csic.es/midas.net/mlis/mlishome.html $>$ ): La Société de l'information multilingue est un programme européen qui s'occupe de promouvoir la diversité linguistique dans la Communauté Européenne. Avec un calendrier prévu pour trois ans (1996-1999), un de ses objectifs est de sensibiliser et d'encourager les services multilingues dans la Communauté, grâce aux technologies, aux ressources et aux normes linguistiques. MLIS participe au financement des projets pilotes avec des organismes publics et privés poursuivant des activités multilingues, et stimule la coopération et la coordination des efforts réalisés au niveau international. Ainsi la Commission européenne a-t-elle mis en place des systèmes pratiques de concertation avec les organismes de langue nationale et le secteur privé, avec les diverses organisations européennes dans le domaine de la traduction, avec les éditeurs de dictionnaires, ainsi que d'autres secteurs pour lesquels le multilinguisme est un élément clé. De plus, MLIS maintient des contacts avec d'autres programmes d'ingénierie linguistique (RDT) ou des programmes de multimédia, tels que INFO2000 ( $<$ http://www2.echo.lu/info2000.fr/infohome.html $>$ ). Il s'agit d'une référence importante pour les services de traduction et d'interprétation des institutions communautaires en tant qu'espace priviligié pour coordonner et adopter les innovations en matière de méthodes, d'outils et de ressources. Dans l'esprit de coopération, cette société a établi des liens avec les organismes européens et internationaux de normalisation tels que le CEN et l'ISO, en ce qui concerne les normes linguistiques, en favorisant leur élaboration dans de nouveaux domaines. MLIS est chargé de promouvoir l'application des normes, telles que UNICODE pour le codage de caractères, l'utilisation de méthodologies pour la création terminologique et l'application de normes ISO 9000 à l'industrie de la langue.

\section{a.2. Projet Interval}

(<http://www.mcs.survey.ac.uk/Research/CS/AI/NEW $>$ ). Il s'agit d'un projet européen de validation de ressources terminologiques dont la durée prévue était de deux ans (1996-1998). Le but visé est l'élaboration de méthodologies et d'outils terminologiques ainsi que leur diffusion et leur validation afin de réduire le coût de la création terminologique; il s'agit aussi d'assurer une meilleure compatibilité entre les différents systèmes de gestion terminologique et d'établir le point de départ de méthodes normalisées. Ce projet a été mis en place avec l'accord des entreprises europénnes de systèmes de gestion terminologique les plus importantes et d'un réseau d'experts en linguistique dans le but d'élaborer des règles et des méthodes pour la création de nouvelles terminologies et d'établir de normes pour adapter les ressources existantes.

a.3. EAGLES: Expert Advisory Group on Language Engineering Standards

(<http://www.ilc.pi.cnr.it/EAGLES/home.html>). Groupe européen qui a entamé ses activités en 1993, dans le cadre d'un programme de la Commission européenne concernant l'ingénierie linguistique. L'objectif du groupe EAGLES est d'encourager et de promouvoir l'élaboration de recommandations et de directives relatives aux ressources linguistiques, aux outils informatiques pour le traitement des données et pour leur évaluation. L'initiative EAGLES contribue à accélérer l'approvisionnement de normes en soulignant les besoins de normalisation dans le domaine de l'industrie de la langue.

b) Projet REALITER (<http://www.iula.upf.es/cpt/cptca.htm $>$ ). Il s'agit d'un réseau panlatin de terminologie qui agit dans le domaine de la terminologie latine (français, espagnol, portugais, italien, catalan et roumain). L'objectif du projet REALITER est l'établissement de méthodes communes et l'élaboration d'outils dans le but de développer les langues néolatines et de collaborer dans la réalisation de travaux terminologiques multilingues.

c) WG Multilingualism (<http://www.dragoman.org/ec $>$ ). Groupe de travail sous l'égide de la DGIII de la Commission européenne qui s'applique à l'élaboration de recomman- 
dations concernant l'utilisation d'outils de traduction multilingues dans le commerce électronique. Il est spécialement adressé aux PME (Petites et moyennes entreprises).

d) Consortium de la World Wide Web (W3C) (<http://www.w3.org/Consortium $>$ ). Le Consortium est une association internationale qui a été créée en 1994 et dont le siège est l'Institute of Technology Laboratory for Computer Science de Massachussetts aux États-Unis, l'Institut national de recherche en informatique et en automatique, en Europe, et la Keio University Shoran Fujisawwa Campus, au Japon. Il travaille sur le développement de protocoles et de formats communs afin d'assurer l'interopérabilité mondiale du réseau, et de réduire les difficultés soulevées par la pluralité des langues et par des systèmes d'écriture différents. Dans ce sens, il définit des modèles et des recommandations pour l'internationalisation du réseau.

\subsection{Ressources électroniques de normalisation}

Le réseau Internet constitue un excellent outil pour la diffusion de l'information sur les normes non seulement pour les traducteurs, mais aussi pour les professionnels de n'importe quel domaine à la recherche d'une norme spécifique.

On trouvera ci-dessous les adresses des pages Web qui fournissent un bon nombre de liens intéressants concernant la normalisation et l'information sur les normes. Il faut ajouter les pages Web des organisations nationales, régionales et internationales.

a) LISA (<http://www.lisa.org $>$ ) : il s'agit de l'URL de LISA (Localisation Industry Standards Association), une organisation non-gouvernamentale et privée qui a été fondée en Suisse en 1990. LISA coordonne les travaux et les efforts réalisés dans le domaine de l'industrie de la localisation et de l'internationalisation. Encourager l'utilisation des normes dans l'élaboration de logiciels multilingues et d'autres produits informatisés constitue l'un de ses objectifs.

b) Internet Standards Ressources $<$ http://silk.tisi.go.th/other.html $>$ : page qui contient des liens d'organisations nationales et internationales de normalisation, et d'autres liens relatifs aux normes et à la normalisation. Elle fournit aussi des informations sur les normes TISI élaborées par l'Organisation thaillandaise de normalisation.

c) Institut of Translation and Interpreting (ITI) <http://www.ITI.org.uk>: il s'agit de l'adresse électronique de l'Institute of Translation and Interpreting (ITI), fondé en 1986 et associé britannique de la Fédération internationale de traducteurs (FIT). Son objectif vise la diffusion des normes de qualité pour la traduction et l'interprétariat dans les domaines de la littérature, des sciences, de la recherche, du commerce, de l'industrie, du droit et de l'administration. L'ITI est devenu une des premières sources d'information de son pays, utilisée par le gouvernement, l'industrie, les médias et par tous ceux qui s'intéressent à la traduction et à l'interprétariat.

d) Université Laval <http://www.bibl.ulaval.ca/ress/normes3.html>: page de ressources sur les normes élaborées par l'Université Laval au Canada, et adressée aux étudiants et aux chercheurs. Elle fournit l'accès aux œuvres et aux références bibliographiques sur la normalisation de sa bibliothèque, l'accès aux bases des données statistiques, l'accès aux catalogues des organisations de normalisation au Canada et dans d'autres pays.

e) RIOFIL < http://www.riofil.org/normalisation.htm>: page qui offre un dossier sur la normalisation présentant une analyse des normes, recommandations, standards et spécifications techniques dans le domaine du traitement des langues en informatique, surtout dans le domaine francophone. En outre, elle popose un grand nombre de liens vers des organismes internationaux de normalisation, ainsi que d'autres ressources normatives. 
f) Institute of Linguists (IOL) <http://www.iol.org.uk $>$ : association internationale fondée en 1910 et dont le siège est à Londres. Avec plus de 7000 associés issus des domaines de la linguistique et de la traduction, ses objectifs visent à améliorer le statut professionnel des linguistes et des traducteurs par le biais d'un "Code de Conduite», qui rassemble des recommandations et des conseils pour atteindre un travail de qualité. Son site Web est une excellente source d'information.

\section{Conclusion}

Les normes élaborées par les organisations de normalisation sont adoptées de plus en plus par les services de traduction et les traducteurs dans le but de garantir la meilleure qualité dans leurs services et dans leurs produits. La normalisation devient donc synonyme de qualité pour les clients qui souhaitent les meilleurs résultats.

D’autre part, les normes représentent aussi un outil indispensable pour les traducteurs, dans la mesure où elles visent à créer et à établir des ressources linguistiques, terminologiques et méthodologiques normalisées afin d'améliorer les échanges nationaux et internationaux, ainsi que la coopération dans toutes les matières. Il faut ajouter à cela les efforts de normalisation dans le domaine des technologies de l'information, surtout celles qui concernent le traitement informatisé des données multilingues ainsi que les autoroutes de l'information et Internet.

La normalisation est présente au cours des procès de la traduction, depuis la régularisation du contrat entre le client et le traducteur jusqu'à la présentation du texte meta. De ce fait, l'accès à l'information normative représente une phase incontournable dans l'activité des traducteurs. Dans cette tâche, ils sont assistés non seulement par de centres de documentation chargés de diffuser l'information des normes élaborées par les organismes nationaux et internationaux, mais aussi d'autres agences privées spécialisées qui se sont spécialisées pour conseiller les clients en matière de normes. De même, le réseau Internet devient un moyen pour accéder à des sites Web qui fournissent l'information nécessaire sur les normes, ainsi que sur les projets de recherche en cours et dont les objectifs sont le développement des normes et des recommandations dans le domaine de l'industrie de la langue.

\section{NOTES}

1. ATIO est une association canadienne fondée en 1921 et qui compte plus de 1000 membres parmi les traducteurs, les interprètes et les terminologues tant du service public que privé.

2. Le Lexique de la norme générale de classification a été créé de concert avec le Secrétariat du Conseil du Trésor dans le cadre du nouveau système de classification de la fonction publique canadienne. Ce lexique compte environ 1500 entrées et s'adresse aux ministères et organismes du gouvernement fédéral dans le but d'assurer un niveau de qualité dans la rédaction et la traduction des descriptions de travail.

\section{RÉFÉRENCES}

CEVEIL (Centre d'expertise et de veilles Inforoutes et Langues). Dossier Les normes. <http:// www.ceveil.qc.ca>

ISO (1980): Guide ISO 2. Termes généraux et leurs définitions concernant la normalisation et la certification, $3^{\mathrm{e}}$ éd., Genève, ISO.

ISO (1986). Accès à l'information normative. Comment se renseigner ou être informé sur les normes et les règlements techniques à travers le monde, Genève, ISO. 
ISO 2788: 1986. Documentation. Principes directeurs pour l'établissement et le développement de thésaurus monolingues.

ISO 5964: 1985. Documentation. Principes directeurs pour l'établissement et le développement de thésaurus bilingues.

ISO/IEC 9126: 1991. Technologies de l'information. Évaluation des produits logiciels. Caractéristiques de qualité et directives d'utilisation.

«Normalisation documentaire. Le défi de l'électronique». Dossier coordonné par Catherine Mattenet (1999). Documentaliste-Sciences de l'information, 36- 2 / 117-127.

NF Z44-004-1984, équivalente à la norme ISO 214:1976. Recommandations aux auteurs des articles scientifiques et techniques pour la rédaction des résumés.

NF Z 41-004:1979. Présentation des traductions.

Bourbeau, L. et F. Pinard, "Normalisation et internationalisation, Inventaire et prospective des normes clefs pour le traitement informatique du français», version finale (@ ACCT- RIOFIL - OQIL), Montréal le 15 octobre 1995, <http://www.ceveil.qc.ca/Normes/index.html>.

Hurtado Albir, A. (ed.) (1996), «La enseñanza de la traducción», Castellón: Universidad Jaume I.

Peña, S. y Maa José Hernández Guerrero. (1994): Traductología, Universidad de Málaga.

Pinto, M. (1993). Análisis documental. Fundamentos y procedimientos, $2^{\mathrm{a}}$ ed. rev. y auhm., Madrid, Eudema.

Sturz, W. (1998): «Does the Language Business Need an ISO Alternative", Language International, 10- 5. 